\title{
Effect of Cluster Size Selection on the Throughput of Multi-hop Cooperative Relay
}

\author{
Sam Vakil ${ }^{\dagger}$, Min Dong ${ }^{\ddagger}$ and Ben Liang ${ }^{\dagger}$ \\ $\dagger$ Department of Electrical and Computer Engineering, University of Toronto, Canada \\ $\ddagger$ Faculty of Engineering and Applied Science, University of Ontario Institute of Technology, Canada. \\ †Email: \{vakil, liang\}@ comm.utoronto.ca, ${ }^{\ddagger}$ Email: min.dong@uoit.ca
}

\begin{abstract}
We study the effect of relay cluster selection on throughout in multi-hop cooperative communications with one source and one destination. We evaluate the effective relay throughput as a function of the source transmission rate and the network outage probability. Assuming channel side information (CSI) only available at the receivers, we formulate the cluster size optimization to maximize throughput. Furthermore, since the bottleneck of the multi-hop relaying is at the first hop where there is in general a lack of cooperation from the source, for the scenario where CSI is available at the source, we may incorporate opportunistic relay selection at the first hop in the cluster optimization problem. Our results demonstrate how the optimal selection of cluster sizes can significantly increase the relaying throughput.
\end{abstract}

\section{INTRODUCTION}

Multi-hop relaying improves coverage and throughput, especially for large-scale extended networks [1]. Its application arises in various type of networks, including ad hoc networks, cellular networks, and vehicular networks. The benefit of usercooperation has been discovered from a different prospective in the form of physical layer cooperative diversity, as a means to combat fading [3], [4]. The problem has its roots in the two-hop relaying, and it is shown that the relay node can be used to improve signal reception reliability. The finding has generated many interests in further analyzing the cooperative gain in different variations of two-hop relay channels and how to realize it with practical schemes [5], [6].

The potential of improved physical layer reliability using cooperative diversity is especially appealing in multi-hop relaying, where the potential packet loss is magnified by the possibility of link failure over each hop. Most previous works use clustering to achieve scalable routing or efficient energy consumption (in sensor networks) at network layer, with simplified assumptions on physical layer processing. If cooperative diversity gain can be carefully incorporated, the multi-hop relaying will not only enjoys more reliable performance but also results in potentially much higher throughput. In this context, coding strategies exploiting node cooperation were developed for multiple relays in [7] as the first information theoretic approach towards cooperative multi-hop relaying. However, it remains an open problem to quantify the capacity of multi-hop cooperative communication. In [8], the authors took a step forward to study the scaling behavior of achievable rate in a network combining distributed

This work was funded in part by an NSERC Strategic Project Grant STPSC 356786-07 and an NSERC Discovery Grant RGPIN 372059-09.
MIMO transmission with multi-hop relaying with clusters. Multi-hop cooperative broadcasting/flooding is studied in an asymptotic regime [9]. Cluster-based multi-hop sensor network is proposed [10] to exploiting MIMO diversity using clustered nodes with fixed cluster size. Most existing work either consider single hop multiple relays, or multi-hop relay clusters with fixed size. However, the cluster selection, including the cluster size and number of hops, is critical to maximize the physical layer cooperative gain and overall data rate. To our best knowledge, this issue has not been studied and little is known on the effect of cluster size on the multi-hop relay performance. This paper presents a step towards this direction.

We study the effect of relay cluster size selection on the end-to-end data rate in a multi-hop cooperative communication setting with single source and destination. The relay nodes employ the decode-and-forward (DF) relaying strategy, and are grouped into clusters to pass source message to the destination. The channel over each communication link undergoes slow fading, and therefore subjects to outage. Assuming channel side information (CSI) is only available at the receivers, we formulate the cluster size optimization as a rate maximization problem.

Constraining on each cluster having the same successful decoding probability, we provide a simple suboptimal solution. For the general optimization problem, we then present a twoloop iterative procedure based on simulated annealing [11] to search for the optimal solution. Our results demonstrate how the optimal selection of cluster sizes can significantly increase the end-to-end data rate. In addition, since the bottleneck of the multi-hop relaying is at the first hop where there is in general a lack of cooperation from the source, we propose to incorporate opportunistic relay selection at the first hop in the cluster size optimization, assuming CSI is available at the source.

\section{PRoblem Formulation}

\section{A. Network Model}

We consider a scenario where a source node $S$ transmits its message to a destination node $D$ through $N$ relay nodes using multi-hop relaying. These $N$ relay nodes can be randomly located between $S$ and $D$. We assume $S$ is located at the origin, and denote the location of relay node $i$ by $\left(x_{i}, y_{i}\right)$, for $i=$ $1, \cdots, N$. The relay nodes are indexed based on the increasing order of their distance from the source $S$, i.e. $d_{S, i} \leq d_{S, i+1}$, where $d_{S, i}=\sqrt{x_{i}^{2}+y_{i}^{2}}$ is the distance between $S$ and node 


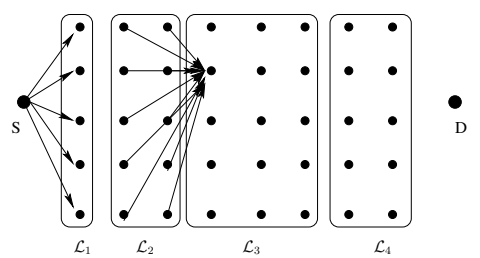

Fig. 1. Schematic of a sample network with one source-destination pair and $N$ relay nodes.

$i$. Similarly, the distance between the relay node $i$ and $j$ is denoted by $d_{i, j}$.

We constrain ourselves to half-duplex transmission, where a relay node is either in transmission or in reception but not simultaneously. Furthermore, we employ a DF relaying strategy, where each relay node decodes, re-encodes, and retransmits the source message. In a $K$-hop relaying scenario, the $N$ relay nodes are grouped into $K$ clusters according to certain selection criterion. The grouping can be represented by $\left\{\mathcal{L}_{1}, \cdots, \mathcal{L}_{K}\right\}$, where $\mathcal{L}_{k}$ is the index set containing the index of those nodes in the $k$ th cluster, $k=1, \cdots, K$. As jointly encoding and decoding within a cluster can be practically challenging, we consider a simple cooperation where, in the $k$ th hop, each node in the $k$ th cluster receive the source message relayed from nodes in the $(k-1)$ th cluster, and try to individually decode, reencodes and retransmit the message. Each node than transmits the message using an orthogonal transmission (e.g. frequency, time, or code).

With proper combining of received signals, the use of multiple nodes in a cluster to relay the same message results in increased received signal power at each receive node, thus improves decoding performance. Fig. 1 shows an example of such multi-hop DF relay scenario.

\section{B. Channel Model}

We assume a slow Rayleigh flat fading channel model, where the channel $h_{i, j}$ is constant over the duration of the message transmission. The received observation at a given node $j$ in the $k$ th cluster from the $(k-1)$ th cluster is given by $y_{i j, k}[n]=$ $h_{i, j} s_{i, k-1}[n]+z_{j}[n]$, where the channel $h_{i, j} \sim \mathcal{C N}\left(0, \sigma_{h}^{2}\right)$ captures path-loss, shadowing, and small-scale flat fading. Its amplitude $\alpha_{i, j}=\left|h_{i, j}\right|$ is Rayleigh distributed with power $E\left[\alpha_{i, j}^{2}\right]=\sigma_{h}^{2}=A d_{i, j}^{-\kappa}$, where $A$ is the path-loss constant; we assume $h_{i, j}$ 's are independent for any $i \in \mathcal{L}_{k-1}$ and $j \in \mathcal{L}_{k}$ pair. We assume all nodes have the same transmission power $P$. The transmitted signal from node $i$ in the $(k-1)$ th cluster is denoted as $s_{i, k-1}[n]$. Since all nodes transmit the same message, $s_{i, k-1}[n]$ is the same for all $i \in \mathcal{L}_{k-1}$. The additive noise at each node $j$ is denoted by $z_{j}[n]$ with variance $\sigma_{z}^{2}$.

\section{Multi-hop Relay Performance}

Assuming the source $S$ transmits its message with rate $C_{o}$, we measure the performance of the multi-hop relaying by the outage probability based on this given transmission rate. Note that the choice of the clustering scheme can significantly affect the outage probability. For a transmission rate $C_{o}$, we denote the probability of correct decoding at node $j \in \mathcal{L}_{k}$ by
$\mathrm{P}_{\mathbf{c}_{j, k}}\left(C_{o}, \mathbf{d}_{j, k}, \mathcal{L}_{k-1}\right)$, where $\mathbf{d}_{j, k}$ is a vector of the distance between any node in the previous $(k-1)$ th cluster to node $j: \mathbf{d}_{j, k}=\left[d_{i_{1}, j}, \cdots, d_{i_{\mid \mathcal{L}_{k-1}, j}}\right]$ and $\mathcal{L}_{k-1}=\left\{i_{1}, \cdots, i_{\left|\mathcal{L}_{k-1}\right|}\right\}$. We define the cluster decoding probability $\mathrm{P}_{\mathrm{c}_{k}}$, for cluster $k$ as the probability that all relays in the cluster decode their received message correctly. Hence, it can be expressed as

$$
\mathrm{P}_{\mathrm{c}_{k}}\left(C_{o}, \mathbf{D}_{k}, \mathcal{L}_{k}\right)=\prod_{j=1}^{\left|\mathcal{L}_{k}\right|} \mathrm{P}_{\mathrm{c}_{j, k}}\left(C_{o}, \mathbf{d}_{j, k}, \mathcal{L}_{k-1}\right),
$$

where $\mathbf{D}=\left[\mathbf{d}_{1, k}, \cdots, \mathbf{d}_{\left|\mathcal{L}_{k}\right|, k}\right]$ contains the distances of all the node pairs from the cluster $k-1$ to the cluster $k$. An outage occurs in the relaying between the clusters $(k-1)$ and $k$ with probability given by $\mathrm{P}_{\text {out }_{k}}\left(C_{o}, \mathbf{D}_{k}, \mathcal{L}_{k}\right)=1-\mathrm{P}_{\mathrm{c}_{k}}\left(C_{o}, \mathbf{D}_{k}, \mathcal{L}_{k}\right)$. Note that this definition of outage is based on our assumption that successful relaying between two clusters is defined as all receiving nodes can decode the message. Although in practise such constraint is not necessary for the message to be relayed from hop to hop, it does, heuristically, pose a criterion for cluster grouping to select those potentially most cooperative relay nodes. In other words, the clustering should prevent including 'non-cooperative' relay node that has low probability to decode message at current hop (thus render itself to be useless). To achieve this, we essentially require the probability of successful decoding for all nodes in the cluster to be higher than a threshold (as we will see in Section III). In addition, this constraint also helps reduce the search space for cluster selection significantly. The effective throughput achieved by multi-hop cooperative relaying now can be written as

$$
R=\frac{C_{o}}{K+1} \prod_{k=1}^{K+1} \mathrm{P}_{\mathrm{c}_{k}}\left(C_{o}, \mathbf{D}_{k}, \mathcal{L}_{k}\right)
$$

where $K$ represents the total number of clusters and $\mathrm{P}_{\mathrm{c}_{K+1}}\left(C_{o}, \mathbf{D}_{K+1}, \mathcal{L}_{K+1}\right)$ is the decoding probability at the destination.

From (2), we see that, to maximize the rate $R$, ideally, we want the successful cluster decoding probability $\mathrm{P}_{\mathrm{c}_{k}}\left(C_{o}, \mathbf{D}_{k}, \mathcal{L}_{k}\right)$ as close to 1 as possible and, at the same time, $K$ as small as possible. However, the relation of two factors are involved: having a smaller hop count $K$ is equivalent to having a larger cluster size for each relay cluster, and thus reduces $\mathrm{P}_{\mathrm{C}_{k}}\left(C_{o}, \mathbf{D}_{k}, \mathcal{L}_{k}\right)$ if the individual node decoding probability is the same. On the other hand, a receiving node will benefit from a bigger transmitting cluster due to cooperative gain, and will have improved decoding probability. Therefore, a trade-off exists between the cluster size and the relay hop counts. Our goal is to find the optimal cluster sizes to maximize the relay throughput $R$. We can express the optimization problem as

$$
\begin{aligned}
& \left(K^{*},\left\{\left|\mathcal{L}_{k}\right|^{*}\right\}_{1}^{K^{*}}\right)=\underset{K,\left\{\left|\mathcal{L}_{k}\right|\right\}_{1}^{K}}{\arg \max } \frac{C_{o}}{K+1} \prod_{k=1}^{K+1} \mathrm{P}_{\mathrm{c}_{k}}\left(C_{o}, \mathbf{D}_{k}, \mathcal{L}_{k}\right) \\
& \text { subject to } \sum_{k=1}^{K}\left|\mathcal{L}_{k}\right|=N .
\end{aligned}
$$

In the following section we elaborate on the cluster size optimization and its effect on the transmission rate. 


\section{Cluster Size Optimization}

In order to find the above optimization solution, we first derive an expression for the cluster decoding probabilities $\mathrm{P}_{\mathbf{c}_{k}}^{*}\left(C_{o}, \mathbf{D}_{k}, \mathcal{L}_{k}\right)$. Then, constraining on all clusters having the same cluster decoding probability threshold, we convert (3) to a simple suboptimal optimization. For the general problem, we then use a two-loop iterative method to search for the optimal cluster size.

\section{A. Evaluating Decoding Probability at Each Relay Node}

Since the relay nodes in a cluster transmit the re-encoded message through orthogonal transmissions, at a received node, the maximum ratio combining technique can be used to add coherently the received signal observations. Then, the received message at node $j$ in the $k$ th cluster can be correctly decoded if

$$
\frac{1}{\left|\mathcal{L}_{k-1}\right|} \log _{2}\left(1+S N R \sum_{m=1}^{\left|\mathcal{L}_{k-1}\right|} \alpha_{i_{m}, j}^{2}\right)>C_{o} .
$$

where $S N R \triangleq P / \sigma_{z}^{2}$ and $P$ is the transmission power at each node. Node indexes in the $(k-1)$ th cluster is given by $\mathcal{L}_{k-1}=$ $\left\{i_{1}, \cdots, i_{\left|\mathcal{L}_{k-1}\right|}\right\}$. The probability of successful decoding is given by

$$
\mathrm{P}_{\mathrm{c}_{k, j}}\left(C_{o}, \mathbf{d}_{j, k}, \mathcal{L}_{k-1}\right)=\mathrm{P}\left(\sum_{m=1}^{\left|\mathcal{L}_{k-1}\right|} \alpha_{i_{m}, j}^{2}>B\left(C_{o}\right)\right),
$$

where $B\left(C_{o}\right)=\left(2^{\left|\mathcal{L}_{k-1}\right| C_{o}}-1\right) / S N R$. The distribution of the combined channel power gain $\sum_{m=1}^{\left|\mathcal{L}_{k-1}\right|} \alpha_{i_{m}, j}^{2}$ can be obtained based on the independent Rayleigh fading assumption. Each channel power gain $\alpha_{i_{m}, j}^{2}$ has exponential distribution with mean $A d_{i_{m}, j}^{-\kappa}: f_{\alpha_{i_{m}, j}^{2}}(x)=\frac{1}{A d_{i_{m}, j}^{-\kappa}} \exp \left(-\frac{x}{A d_{i_{m}, j}^{-\kappa}}\right)$ for $x>0$ and 0 otherwise.

From the source $S$ to the first relay cluster, since $\left|\mathcal{L}_{0}\right|=1$, we have $\mathrm{P}\left(\alpha_{S, j}^{2}>B\left(C_{o}\right)\right)=\exp \left(-B\left(C_{0}\right) \frac{d_{S, j}^{\kappa}}{A}\right)$. In general, since the distance $d_{i_{m}, j}$ is different for different node in $\mathcal{L}_{k-1}$, the distributions $f_{\alpha_{i_{m}, j}^{2}}(x)$ are not identical, and it is intractable to derive a closed form for $\mathrm{P}_{\mathrm{c}_{j, k}}\left(C_{o}, \mathbf{d}_{j}, \mathcal{L}_{k-1}\right)$. Instead, we use Lyapunov's central limit theorem [12] to approximate $\mathrm{P}_{\mathbf{c}_{j, k}}\left(C_{o}, \mathbf{d}_{j, k}, \mathcal{L}_{k-1}\right)$ for $k>1$.

Since $\alpha_{i j}^{2}$ is an exponential random variable with mean $A d_{i j}^{-\kappa}$, and variance $A^{2} d_{i j}^{-2 \kappa}$. We can show that the sum $\sum_{m=1}^{\left|\mathcal{L}_{k-1}\right|} \alpha_{i_{m}, j}^{2}$ satisfies the Lyapunov condition, and its normized form can be approximated as Gaussian random variable. Therefore, as the number of relay nodes $N$ increases and the cluster size $\left|\mathcal{L}_{k}\right|$ increases, our approximation becomes more accurate.

Based on the above approximation, the probability of correct decoding can be written as

$$
\begin{aligned}
& \mathrm{P}_{\mathbf{c}_{j, k}}\left(C_{o}, \mathbf{d}_{j}, \mathcal{L}_{k}\right) \\
& =\mathrm{P}\left(\frac{\sum_{m=1}^{\left|\mathcal{L}_{k-1}\right|}\left(\alpha_{i_{m}, j}^{2}-A d_{i_{m}, j}^{-\kappa}\right)}{\left(\sum_{m=1}^{\left|\mathcal{L}_{k-1}\right|} A^{2} d_{i_{m}, j}^{-2 \kappa}\right)^{1 / 2}}>\frac{B\left(C_{o}\right)-\sum_{m=1}^{\left|\mathcal{L}_{k-1}\right|} A d_{i_{m}, j}^{-\kappa}}{\left(\sum_{m=1}^{\left|\mathcal{L}_{k-1}\right|} A^{2} d_{i_{m}, j}^{-2 \kappa}\right)^{1 / 2}}\right) \\
& =1-Q\left(\frac{\sum_{m=1}^{\left|\mathcal{L}_{k-1}\right|} A d_{i_{m}, j}^{-\kappa}-B\left(C_{o}\right)}{\left(\sum_{m=1}^{\left|\mathcal{L}_{k-1}\right|} A^{2} d_{i_{m}, j}^{-2 \kappa}\right)^{1 / 2}}\right)
\end{aligned}
$$

where $Q(\cdot)$ is the Q-function.

\section{B. Iterative Calculation of Cluster Sizes}

The optimization in (3) is a non-convex integer programming problem. We resort to numerical optimization to find the solution, and will demonstrate the numerical results of this optimization in Section IV.

In order to search for the solution, we now use a different angle to look at the optimization in (3). The vector $\mathrm{P}_{\mathrm{c}}^{*}=\left[\mathrm{P}_{\mathrm{C}_{1}}^{*}, \cdots, \mathrm{P}_{\mathrm{C}_{K^{*}}}^{*}\right]$ contains the optimal cluster decoding probability of the message. It is the result of the optimization in (3). Given the optimal cluster decoding probability, we want to find the minimum number of clusters $K^{*}$ so that the throughput $R$ is maximized. We want to form the $k$ th cluster to include as much nodes as possible so long as the cluster decoding probability is no less than the optimal one. Thus, conditioned on knowing $\mathrm{P}_{\mathrm{c}}^{*}$, each optimal cluster size can be quantified as

$$
\begin{array}{ll} 
& \left|\mathcal{L}_{k}\right|^{*}=\max \left|\mathcal{L}_{k}\right| \quad \forall \quad 1 \leq k \leq K^{*} \\
\text { s.t. } & \prod_{m=1}^{\left|\mathcal{L}_{k}\right|} \mathrm{P}_{\mathbf{c}_{j_{m}, k}}^{*}\left(C_{o}, \mathbf{d}_{j_{m}, k}, \mathcal{L}_{k-1}\right) \geq \mathrm{P}_{\mathbf{c}_{k}}^{*} \\
\text { and } \quad & j_{m} \notin \bigcup_{i=1}^{k-1} \mathcal{L}_{i}, \quad \sum_{i=1}^{k}\left|\mathcal{L}_{i}\right| \leq N .
\end{array}
$$

To solve the original optimization in (3), we need to search for the optimal cluster decoding probability vector $\mathrm{P}_{c}^{*}$, and then the optimal cluster size under $\mathrm{P}_{c}^{*}$. Since finding $\mathrm{P}_{c}^{*}$ also depends on the size of clusters, we use iterative method to the above procedure to find the solution.

In the following, we first consider optimizing the cluster size in a subspace of the original optimization problem by constraining all clusters having the same optimal cluster decoding probability; then in the second part, we use heuristic iterative method to search for the optimal cluster size.

1) Fixed threshold for cluster decoding probability $P_{c_{k}}$ : We first consider the optimization in a simplified problem where each cluster decoding probability is constrained to be equal. In particular, we assume $\mathrm{P}_{\mathbf{C}_{k}}^{*}=\mathrm{P}_{\text {th }}$ for all clusters. Under the above simplified assumptions, the optimization problem can be expressed as

$$
\left(K^{*},\left\{\left|\mathcal{L}_{k}\right|^{*}\right\}_{1}^{K^{*}}, \mathrm{P}_{\mathrm{th}}^{*}\right)=\underset{\mathrm{P}_{\mathrm{th}}, K,\left\{\left|\mathcal{L}_{k}\right|\right\}_{1}^{K}}{\arg \max } \frac{C_{o}}{K+1} \mathrm{P}_{\text {th }}^{K}
$$

$$
\begin{array}{ll}
\text { s.t. } & \text { (a) } \prod_{j=1}^{\left|\mathcal{L}_{1}\right|} e^{-\frac{d_{S, j}^{\kappa} B\left(C_{o}\right)}{A}} \geq \mathrm{P}_{\mathrm{th}} \\
& \text { (b) } \prod_{m=1}^{\left|\mathcal{L}_{k}\right|} \mathrm{P}_{\mathrm{c}_{j_{m}, k}}\left(C_{o}, \mathbf{d}_{j, k}, \mathcal{L}_{k-1}\right) \geq \mathrm{P}_{\mathrm{th}} \quad \text { for } \quad k>1 \\
\text { (c) } \sum_{k=1}^{K}\left|\mathcal{L}_{k}\right|=N .
\end{array}
$$

This optimization problem can be numerically carried out by finding the minimum $K$ for $\mathrm{P}_{\mathrm{th}} \in(0,1)$, and then obtaining the $\left(K^{*}, \mathrm{P}_{\mathrm{th}}^{*}\right)$ that gives the maximum throughput $R$. 
2) Variable threshold for cluster decoding probability $P_{c_{k}}$ : Constraining on the same cluster decoding probability in the previous section leads to a suboptimal but simplified rate maximization problem. For the original optimization problem in (3), we use a heuristic method to find the solution. Since the search space is discrete, we use simulated annealing (SA) [11] to search the global maximum. Since the maximization problem is non-convex, there may be multiple local maxima. Thus, the search consists of inner loop and outer loop iterations. The outer loop is used to set a new initial point and the inner loop is applying the SA iterations to search for a local maxima. The initial starting point at the outer loop is set based on the constraint of using the common threshold $\mathrm{P}_{\text {th }}$ for each cluster decoding probability, described in the previous section. The main procedure is listed below.

1) Initialize $\mathrm{P}_{\mathrm{th}}=0, R_{\max }=0$. Let vector $\mathbf{R}$ be $M \times 1$ initialized by 0 , where $\mathbf{R}$ records the throughput $R$ in the recent $M$ iterations. Set maximum iterations $I_{\max }$.

2) Set $\mathrm{P}_{\mathrm{C}_{k}}^{*}=\mathrm{P}_{\text {th }}$ as the common threshold for the cluster decoding probability for each cluster. Let $T$ be the system temperature. Set $T=T_{o}$. Set iteration counter $i=1$.

3) Solve the cluster size optimization problem in (7), except that $\mathrm{P}_{\text {th }}$ is now fixed, instead of being optimized between $(0,1)$, i.e.,

$$
\left(K^{*},\left\{\left|\mathcal{L}_{k}\right|^{*}\right\}_{1}^{K^{*}}\right)=\underset{K,\left\{\left|\mathcal{L}_{k}\right|\right\}_{1}^{K}}{\arg \max } \frac{C_{o}}{K+1} \mathrm{P}_{\text {th }}^{K}
$$

subjects to the same three conditions given in (7). Obtain $K,|\mathbf{L}| \triangleq\left\{\left|\mathcal{L}_{k}\right|\right\}_{1}^{K}$, and $R$. Let $K^{*}=K,|\mathbf{L}|^{*}=|\mathbf{L}|$, and $R_{\max }=R$, respectively.

4) Let $\mathrm{P}_{\mathrm{c}_{k}}^{* \text { old }}=\mathrm{P}_{\mathrm{c}_{k}}^{*}$. Add random perturbations to $\mathrm{P}_{\mathrm{c}_{k}}^{*}$. Solve the optimization in (6) to obtain $K^{\text {new }},\left\{\left|\mathcal{L}_{k}\right|^{\text {new }}\right\}$. If more than $K^{*}$ clusters are needed in the optimization, pad $\mathrm{P}_{\mathrm{C}_{k}}^{*}=\mathrm{P}_{\mathrm{th}}$, for $k>K^{*}$. Derive the rate $R$ using (2).

a) If $R>R_{\max }$ : Let $R_{\max }=R,|\mathbf{L}|^{*}=|\mathbf{L}|^{\text {new }}$ and hop count $K^{*}=K^{\text {new }}$. Left shift values in $\mathbf{R}$ by 1 , and add $R$ to the end of $\mathbf{R}$.

b) Otherwise, we are in a "bad" state: Let $\Delta R=R^{*}-$ $R$.

i. With probability $1-e^{-\frac{\Delta R}{T}}$, let $\mathrm{P}_{\mathrm{c}_{k}}^{*}=\mathrm{P}_{\mathrm{C}_{k}}^{* \text { (old) }}$, i.e., return to the previous cluster decoding probability threshold. No change for $K^{*}$ and $|\mathcal{L}|^{*}$.

ii. With probability $e^{-\frac{\Delta R}{T}}$, accept "bad" state, and update $R_{\max }=R,|\mathbf{L}|^{*}=|\mathbf{L}|^{\text {new }}$ and hop count $K^{*}=K^{\text {new }}$. Left shift values in $\mathbf{R}$ by 1 , and add $R$ in the end of $\mathbf{R}$.

5) Compute average rate $\bar{R}=\operatorname{mean}(\mathbf{R})$. Repeat steps $3-5$ until $\left|R_{\max }-\bar{R}\right|<\delta$.

6) Let $i=i+1$. If $i \leq I_{\max }$, decrease $T: T=T-\Delta T$ and repeat 3-6.

7) Store the $R_{\max }$ obtained from this iteration. While $\mathrm{P}_{\text {th }}<$ 1 , let $\mathrm{P}_{\text {th }}=\mathrm{P}_{\text {th }}+\Delta \mathrm{P}_{\text {th }}$, and repeat steps 2-7.

8) Compare $R$ 's obtained from each outer loop iteration, and find the optimal solution.
The vector $\mathrm{P}_{\mathrm{c}}^{*}$ is a close approximation [11] to the optimal cluster decoding probabilities that give the maximum throughput to the optimization problem. As we will see in Section IV, the simpler suboptimal solution in Section III-B1 with common $\mathrm{P}_{\mathrm{c}, k}^{*}$ can be a good approximation to the general optimization problem.

\section{NuMERICAL RESUlts}

For numerical illustration, we consider a rectangular network topology as shown in Fig. 1, where nodes are placed in $M$ rows and $K_{\max }$ columns with equal column distance. The total number of nodes is $N=M K_{\max }$. We assume the distance between the source and the destination is $d_{S D}=11 \mathrm{~m}$, and the horizontal separation of the relay nodes equals $d=1 \mathrm{~m}$. Hence, the number of relay nodes sharing the same horizontal coordinate follows $M=N \frac{d}{d_{S D}-d}$. A path loss exponent of $\kappa=4$ is considered. The relay nodes have a common transmit power resulting $S N R=P / \sigma_{z}^{2}=10 \mathrm{~dB}$.

We first study the suboptimal solution that uses a common decoding threshold in all the clusters as in (7), assuming $N=$ 50, 100, 200. In Fig. 2, we plot the effective throughput $R$ at the destination computed in (2) vs. the source transmission rate $C_{o}$. For each $N$, the plot shows that the achieved throughput first increases with increased transmission rate $C_{o}$, then decreases with it. This shows that if the sender transmits at a lower bitrate than the optimal value, $C_{o}^{*}$, the cooperative gain is not fully exploited. On the other hand, if the transmission rate exceeds the optimal $C_{o}^{*}$, the outage probability at each relay node increases, leading to deteriorated system throughput.

For the case where near-optimal decoding threshold probabilities are determined using simulated annealing, we plot the effective throughput vs. the source transmission rate in Fig. 3. For each $N$, similar to Fig. 2, the general trend for $R$ is first increasing and then decreasing. More importantly, we note that the main performance metric of interest in many systems is the maximum achievable throughput by optimizing the transmission rate $C_{o}$. In this regard, by comparing the peak values of each plot in these two figures, we observe that the common-threshold simplification achieves between $80 \%$ to $90 \%$ of the maximum achievable throughput by simulated annealing.

For baseline comparison, we compute using (2) the maximum achievable throughput for two naive relaying alternatives. In the first alternative, a single relay in each column is chosen to participate in node-by-node forwarding. The maximum throughput in this case is $0.0106 \mathrm{bits} / \mathrm{s} / \mathrm{Hz}$. In the second alternative, all $M$ relays in each column form a cluster. The maximum throughput in this case is $0.014,0.017$, and 0.019 bits/s/Hz for $N=50,100,200$, respectively. In comparison with the peak throughput shown in Figures 2 and 3, the above demonstrates the significant advantage of optimizing the cluster sizes in multi-hop cooperative relaying.

Furthermore, we have observed that in all cases, the optimal cluster size for each hop first increases and then decreases, as the message is relayed from the source to the destination. Clearly, since the earlier and later hops benefit less from the 


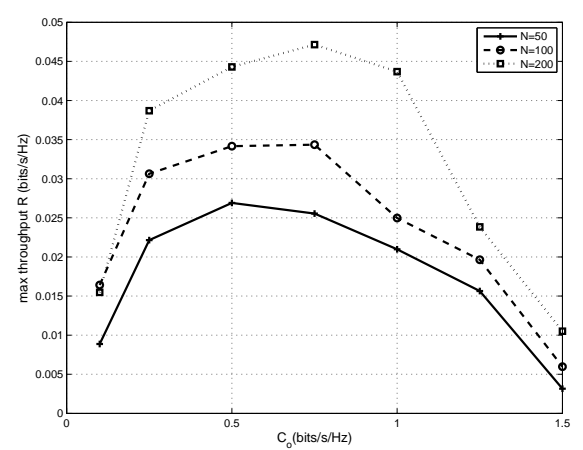

Fig. 2. Throughput vs. transmission rate. Common decoding threshold probability at the clusters.

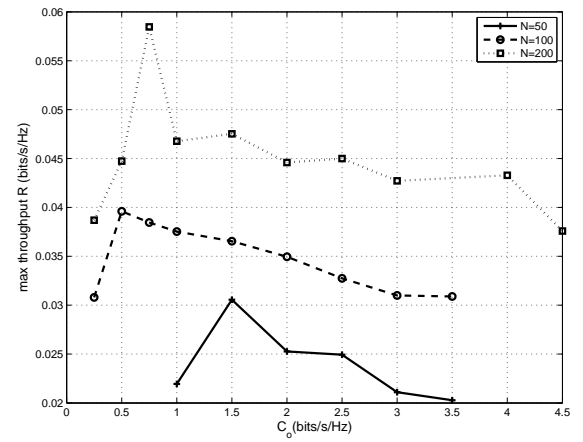

Fig. 3. Throughput vs. transmission rate. Optimal decoding threshold probabilities by simulated annealing.

cooperation of multiple nodes, a smaller cluster size is needed there to maintain an acceptable cluster decoding probability. The figure is omitted due to page limitation. Next, we briefly discuss an opportunistic relaying method for the first hop, which alleviates this problem.

\section{FiRST-HOp OpPoRtunistic RELAy SELECTION}

In multi-hop cooperative relaying, the first hop is clearly the communication bottleneck. This is due to the fact that the source $S$ is the only transmitting node, and therefore relaying does not benefit from cooperation of multiple nodes. The first hop can severely limit the performance gain attained via cooperation. A natural way to increase the multi-hop communication rate is to harness the fading diversity gain in the first hop. In (6), the decoding probability for each relay is derived based on the fading statistics. Hence, when CSI is available at the source, by choosing those with "good" channel conditions between the source and relay nodes, both $R$ and the decoding probability $\mathrm{P}_{\mathrm{C}_{S, 1}}^{*}\left(C_{o}, \mathbf{d}_{S, 1},\left|\mathcal{L}_{0}\right|\right)$ would increase.

We denote the channel power gain between two nodes by $g_{i, j}=\alpha_{i, j}^{2}$, and the $m$ th largest random variable among a set of ordered variables by $g_{i j}^{(m)}$. The optimization in (6) can be modified as follows to compute $\left|\mathcal{L}_{1}\right|$ :

$$
\begin{array}{ll} 
& \left|\mathcal{L}_{1}\right|^{*}=\max \left|\mathcal{L}_{1}\right| \\
\text { s.t. } & \left(1-F_{g_{i j}^{(1)}}\left(B\left(C_{o}\right)\right)\right) \cdots\left(1-F_{g_{i j}^{\left(\left|\mathcal{L}_{1}\right|\right)}}\left(B\left(C_{o}\right)\right)\right) \geq \mathrm{P}_{\mathbf{C}_{1}}^{*}
\end{array}
$$

$$
\begin{aligned}
& \prod_{m=1}^{\left|\mathcal{L}_{k}\right|} \mathrm{P}_{\mathbf{c}_{j_{m}, k}}^{*}\left(C_{o}, \mathbf{d}_{j_{m}, k},\left|\mathcal{L}_{k-1}\right|\right) \geq \mathrm{P}_{\mathbf{C}_{k}}^{*} \\
& \text { and } j_{m} \notin \bigcup_{i=1}^{k-1} \mathcal{L}_{i} \text { and } \sum_{l=1}^{k}\left|\mathcal{L}_{l}\right| \leq N \quad \text { for } \quad k>1
\end{aligned}
$$

where $F_{g^{(j)}}(\cdot)$ is the cumulative distribution function of the $j$ th largest channel gain. By adopting such a channel aware communication scheme, the selected relay nodes in the first cluster will have much stronger links towards the source, and the source can therefore transmit at a significant higher rate.

\section{CONCLUSION}

In this work, we have evaluated the effect of cluster-size selection on multi-hop cooperative relaying performance. The DF relay strategy is considered. We show how the choice of optimal cluster sizes could significantly improve the relay throughput. Assuming CSI at the receivers only, we use heuristic and iteration methods to search for the optimal cluster sizes. Moreover, we have proposed incorporating opportunistic relay selection for the first hop in cluster-size selection to further improve the system throughput.

\section{REFERENCES}

[1] P. Gupta and P. R. Kumar, "The capacity of wireless networks," IEEE Trans. Inform. Theory, vol. 46, no. 2, pp. 388-404, March 2000.

[2] L. L. Xie and P. R. Kumar, "On the path-loss attenuation regime for positive cost and linear scaling of transport capacity in wireless networks," Joint Special Issue of IEEE Transactions on Information Theory and IEEE/ACM Transactions on Networking on Networking and Information Theory, vol. 52, no. 6, pp. 2313-2328, June 2006.

[3] A. Sendonaris, E. Erkip, and B. Azhang, "User cooperation diversity part I: System description," IEEE Transactions on Communications, vol. 51, no. 11, pp. 1927-1938, November 2003.

[4] J. N. Laneman, D. N. Tse, and G. W. Wornell, "Cooprative diversity in wireless networks: Efficient protocols and outage behaviour," IEEE Transactions on Information Theory, vol. 50, no. 12, pp. 3062-3080, December 2004.

[5] J. N. Laneman and G. W. Wornell, "Distributed space-time-coded protocols for exploiting cooperative diversity in wireless networks," IEEE Transactions on Information Theory, vol. 49, no. 10, pp. 2415-2425, October 2003.

[6] R. U. Nabar, H. Bolcskei, and F. W. Kneubuhler, "Fading relay channels: Performance limits and space time signal design," IEEE Journal on Selected Areas in Communications, vol. 22, no. 6, pp. 1099-1109, August 2004.

[7] G. Kramer, M. Gastpar, and P. Gupta, "Cooperative strategies and capacity theorems for relay networks," IEEE Transactions on Information Theory, vol. 51, no. 9, pp. 3037-3063, September 2005.

[8] S. Vakil and B. Liang, "Effect of joint cooperation and multi-hopping on the capacity of wireless networks," in Proc. IEEE Communications Society Conference on Sensor, Mesh and Ad Hoc Communications and Networks(SECON), June 2008.

[9] B. S. Mergen, A. Scaglione, and G. Mergen, "Asymptotic analysis of multi-stage cooperative broadcast in wireless networks," IEEE Transactions on Information Theory, vol. 52, no. 6, pp. 2531-2550, June 2006.

[10] A. del Coso, S. Savazzi, U. Spagnolini, and C. Ibars, "Virtual mimo channels in cooperative multi-hop wireless sensor networks," in Proc. 40th Annual Conference on Information Sciences and Systems, March 2006, pp. 75-80.

[11] P. J. M. van Laarhoven and E. H. L. Aart, Simulated Annealing: Theory and Applications. D. Reidel Publishing Company, 1987.

[12] P. Billingsley, Probability and Measure, 2nd ed. John Wiley and Sons, 1986.

[13] A. Bletsas, A. Khisti, D. P. Reed, and A. Lippman, "A simple cooperative diversity method based on network path selection," IEEE Journal on Selected Areas in Communications, vol. 24, no. 3, March 2006. 